WEI, R.-P. and D. LINDGREN (1995): Optimal family contributions and a linear approximation. Theor. Pop. Biol. 48: $318-332$.

WEI, R.-P. and F. C. YEH (2000): Optimal family contributions in selection toward maximum gain under restricted average coancestry. Silvae Genet. 49: 281-290.

WEI, R.-P., F. C. YEH and N. K. DHIR (2002): Investigation of status number following selection from populations under different mating designs. Silvae Genet. 51: 87-92.

Wei, R.-P., C. R. Hansen, N. K. DhiR and F. C. YeH (1998): Genetic gain with desired status number in breeding programs: a study on selection effects. Can. J. For. Res. 28: $1861-1869$
White, T. L. and G. R. Hodge (1988): Best linear prediction of breeding values in a forest tree improvement program. Theor. Appl. Genet. 76: 719-727.

Williams, C. G. and O. SAVolainen (1996): Inbreeding depression in conifers: implications for using selfing as a breeding strategy. For. Sci. 42: 102-117.

WRAY, N. R. and M. E. GODDARD (1994): Increasing longterm response to selection. Genet. Sel. Evol. 26: 431-451.

Zheng, Y. Q., D. Lindgren, O. Rosvall and J. Westin (1997): Combining genetic gain and diversity by considering average coancestry in clonal selection of Norway spruce. Theor. Appl. Genet. 95: 1312-1319.

\title{
Accuracy of Ranking Individuals in Field Tests of Different Designs: A Computer Simulation
}

\author{
By C.-Y. XIE ${ }^{1), *)}$, Y.-B. FU ${ }^{2)}$ and A. D. YANCHUK ${ }^{1)}$
}

(Received $4^{\text {th }}$ October 2005)

\begin{abstract}
A computer simulation was conducted to investigate the accuracy of ranking individual trees in field tests of different designs. A test population that consists of 900 trees from 45 full-sib families generated by three 6-parent disconnected half-diallels was considered. One incomplete block design with single-tree plots and four complete block designs with 1, 2, 4, and 10-tree row plots were examined. Various narrow-sense heritabilities, site variation patterns (patch sizes and gradient slopes), and two levels of dominant to additive genetic variance ratios (0 and 0.30$)$ were evaluated. Results indicate that the accuracy of ranking depends more on the heritability of the trait and less on the magnitude of the dominant genetic variance, site variation patterns, and field designs. With patchy site variation, differences in ranking accuracy were observed for different designs, but became smaller with higher heritabilities. Impact of environmental gradient on the accuracy of individual ranking was negligible. Incomplete block design with single-tree plots (ICB1) provided the most accurate ranking when heritability was low while complete block design with 2-tree plots (RCB2) appeared to be the best when heritability was high. Large row plot designs were among the least effective in all the simulated scenarios. For traits with medium heritabilities, the statistical efficiencies of ICB1 and RCB with 1 and 2-tree plots are comparable.
\end{abstract}

Key words: breeding value, progeny testing, field design, ranking accuracy, computer simulation.

\footnotetext{
1) British Columbia Ministry of Forests, Research Branch, P.O. Box 9519, Victoria, BC V8W 9C2, Canada.

2) Plant Gene Resources of Canada, AAFC Saskatoon Research Centre, 107 Science Place, Saskatoon, SK S7N 0X2, Canada.

*) Corresponding Author: ChANG-YI XIE, Tel: (250) 387-8911, Fax: (250) 387-0046. E-mail: chang-yi.xie@gov.bc.ca.
}

\section{Introduction}

Progeny testing is one of the most important and expensive phases of a tree-breeding program. To make maximum progress from selection, it is necessary to use a field design that ranks the test materials as closely as possible to the ranking of their true genetic merit, or breeding values. Single-tree plot (STP) designs (with either randomized complete or incomplete blocks) have long been recognized as the best for ranking families, or parents, for backward selection (CONKLE, 1963; WRIGHT, 1976; LiBBy and CockerhaM, 1980). As they maximize the number of micro-sites sampled with a given number of trees per family, STP designs minimize the errors in estimating family means (or parental breeding values) and thus maximize the accuracy of family ranking. In tree breeding, forward selection is another common practice, which requires accurate ranking of individuals.

The breeding value of an individual (IBV) consists of two components corresponding to family and withinfamily additive genetic effects. Although STP designs are efficient for evaluating the first component (i.e., parental breeding values), they may not be for the second (i.e., within-family breeding value) since they are unable to provide assessment of plot effects for microsite adjustments to the observed individual phenotypes. Hence STP designs may not always be superior to multiple-tree plot (MTP) designs in ranking individuals, and their relative superiority may vary with the heritability of the trait and the patterns of environmental variation (VAN BUIJTENEN, 1983).

In this paper, we report the results from a computer simulation on the accuracy of ranking individuals in field tests of different designs. This simulation consid- 
ered a wide range of heritability and various scenarios of environmental variation patterns.

\section{Simulation Methods}

A test population that consists of 900 individuals from 45 full-sib families (i.e., 20 individuals per family) generated by three 6-parent disconnected half-diallels was considered in the simulation. Although it is relatively small due to limited computational capability it does represent the typical size of any one phase or a unit (e.g., a subline) of a larger testing program, which may involve the breeding and testing of several dozen selected parents in an advanced generation breeding program.

Simulation was conducted with a computer program written in SAS ${ }^{\circledR}$ MACRO and IML, which consists of the following five steps:

1) Generating a spatial error distribution for the 900 planting spots with specified patch sizes and gradients of environmental variation: A test site with 20 rows by 45 columns was the considered layout on the ground and site variation was modelled according to the method described by Fu et al. (1998). In this study, we considered three levels of patch size $(\alpha=1,6$ and 10) and gradient $\left(\gamma=0,-\tan \left(5^{\circ}\right)\right.$, and $\left.-\tan \left(10^{\circ}\right)\right)$ which represent a typical spread of site variations observed in the Douglas-fir progeny trials on coastal British Columbia (Fu et al., 1999a).

2) Generating genetic values for the 900 individuals with specified additive genetic variance $\left(\sigma_{A}^{2}\right)$ and dominant variance $\left(\sigma_{D}^{2}\right): \sigma_{A}^{2}$ and $\sigma_{D}^{2}$ were assigned by specifying the narrow-sense heritability $\left(h^{2}\right)$ and dominant to additive genetic variance ratio, and setting the total phenotypic variance to an arbitrary value of 100 . In this simulation, five levels of narrow-sense heritability $(0.05$, $0.15,0.25,0.35$ and 0.45 ) and two levels of dominant to additive genetic variance ratio $(0,0.30)$ were used. The total genetic value of an individual was defined as:

$$
G_{k l m}=f_{k}+m_{l}+f m_{k l}+A w_{k l m}+D w_{k l m} .
$$

All effects in the model were considered random with normal independent distribution (NID). $f_{k}$ and $m_{l}$ are the general combining ability (GCA) effects of the $k$ th female and $l$ th male parent $\sim \operatorname{NID}\left(0,1 / 4 \sigma_{A}^{2}\right)$, respectively, $\mathrm{fm}_{k l}$ is the specific combining ability (SCA) effect of the $k l$ th cross (or family), $\sim \operatorname{NID}\left(0,1 / 4 \sigma_{D}^{2}\right), A w_{k l m}$ is the within-family additive genetic effect of the $k l m$ th individual, $\sim \operatorname{NID}\left(0,1 / 2 \sigma_{A}^{2}\right)$, and $D w_{k l m}$ is the within-family dominant genetic effect of the $k l m$ th individual, $\sim \operatorname{NID}\left(0,3 /{ }_{4} \sigma_{D}^{2}\right)$. This model assumes that there is no epistatic effect between involved genes and the diallel effect is zero.

The true breeding value of the individual (IBVt) is the additive genetic component of its total genetic value:

$$
I B V t_{k l m}=f_{k}+m_{l}+A w_{k l m} .
$$

With the specified additive genetic and dominant variances, the variance of each genetic effect in Equation 1 was calculated. The GCA effects for the 18 parents, SCA effects for 45 crosses, and within-family additive and dominant genetic effects for the 900 individuals were generated by using random number generators. First, a set of random numbers (or values) with a standard normal distribution were generated by a random number generator, and then, those random values were multiplied by the standard deviation of the corresponding genetic effect to convert them to have a normal distribution of zero mean and the calculated variance for that genetic effect. The total genetic value $\left(G_{k l m}\right)$ and the true breeding value $\left(I B V t_{k l m}\right)$ of an individual were calculated according to Equations 1 and 2, respectively.

3) Generating the design matrices: Five block designs were examined: i) 60 incomplete blocks of size 15 with single-tree plots (ICB1); ii) 20 complete blocks of size 45 with single-tree plots (RCB1); iii) 10 complete blocks of size 90 with 2-tree row plots (RCB2); iv) five complete blocks of size 180 with 4 -tree row plots (RCB4); and v) two complete blocks of size 450 with 10 -tree row plots (RCB10).

For ICB1, the NC design (i.e., no constraint over replications) with STPs was applied. We chose to use the NC ICB approach as it has been shown to be very close in efficiency to the 'alpha' design (Fu et al., 1998). In this design, the 45 seedlings, one from each of the 45 families, was divided into three blocks of size 15 . For each block, 15 families were randomly assigned without replacement. This was repeated for each replication so that the same 15 families in the first block in the first replication were not allocated into the same block in the next replication. For RCB1, the number of complete blocks is 20 , each consisting of 45 families with one seedling per family. For RCB2, the number of complete blocks is 10 , each consisting of 45 families with two seedlings per family planted in a row (i.e., 2-tree row plot). For RCB4, the number of complete blocks is five, each consisting of 45 families with four seedlings per family planted in a row as a 4-tree row plot. Similarly for RCB10, the number of complete blocks is two, each consisting of 45 families with 10 seedlings per family planted in a row as a 10-tree row plot. In this simulation, a row plot was placed in the column direction (i.e., parallel to the environmental gradient). These design matrices were then combined with the genetic data generated in Step 2 and sorted by block and row (or plot) for randomization in the next step.

4) Generating the phenotypic data: The randomized data were sorted again by block and row before the genetic value was added to the environmental error to generate the phenotypic value of an individual. Two rounds of sorting are required to make row plots match with the physical settings generated in Step 1.

5) Analyzing simulated data and predicting individual tree breeding values: For each combination of input parameters, 1000 independent runs were conducted.

For multiple-tree plot (MTP) designs, analysis of variance (ANOVA) was performed based on the following linear mixed model:

$$
y_{i j k l m}=u+B_{i}+f_{k}+m_{l}+f m_{k l}+p_{i j k l}+w_{i j k l m},
$$

where $u$ is the population mean, $B_{i}$ and $p_{i j k l}$ are the block and plot effects, respectively, and $w_{i j k l m}$ is the error term. The other effects are defined in Equation 1. Except for $B_{i}$, all the other effects were considered ran- 
dom. Variance components for $f, m, f m, p$ and $w$ (i.e., $\hat{\sigma}_{f}^{2}$, $\hat{\sigma}_{m}^{2}, \hat{\sigma}_{f m}^{2}, \hat{\sigma}_{p}^{2}$ and $\hat{\sigma}_{w}^{2}$ ) were estimated using SAS ${ }^{\circledR}$ Proc Mixed with option of REML (SAS INSTITUTE INC., 1995). For single-tree plot (STP) designs (ICB1 and RCB1), plot effect $(p)$ was removed from Equation 3.

The procedure described by XIANG and LI (2003) was employed to predict individual breeding value $(I B V p)$ for each tree:

$$
I B V p=\left[\frac{\hat{\sigma}_{f}^{2}}{\hat{\sigma}_{w}^{2}} \boldsymbol{Z}_{f} \boldsymbol{Z}_{f}+\frac{\hat{\sigma}_{m}^{2}}{\hat{\sigma}_{w}^{2}} \boldsymbol{Z}_{m} \boldsymbol{Z}_{m}+\frac{\left(\hat{\sigma}_{f}^{2}+\hat{\sigma}_{m}^{2}\right)}{\hat{\sigma}_{w}^{2}} \boldsymbol{I}_{n}\right](\boldsymbol{Y}-\boldsymbol{X} \hat{\boldsymbol{\beta}}-\boldsymbol{Z} \hat{\boldsymbol{\gamma}}),
$$

where $\boldsymbol{Z}_{f}$ and $\boldsymbol{Z}_{\boldsymbol{m}}$ are design matrix for female and male parents, respectively, and $(\boldsymbol{Y}-\boldsymbol{X} \hat{\boldsymbol{\beta}}-\boldsymbol{Z} \hat{\boldsymbol{\gamma}})$ is the residual obtained directly from the output of SAS ${ }^{\circledR}$ Proc Mixed.

Kendall's coefficient of correlation (SoKAL and ROHLF, 1981) between ranks of the true and predicted individual breeding values (i.e., $I B V t$ and $I B V p$, respectively) was employed to quantify the accuracy of ranking individuals according to their true genetic merit. The "realized" percent gain from selecting the top 100 individuals based on $I B V p$ was used as another indicator to the effectiveness of the field designs for ranking individuals for forward selection. This was calculated as the percentage of the total true breeding value of the top 100 individuals ranked based on $I B V p$ over the total true breeding value of the top 100 individuals ranked based on IBVt. It is important to clarify that we are not proposing to select the top 100 individuals for future breeding or production. For that purpose, both the breeding values and the relatedness of the selection candidates should be considered and the final decision should be made based on the group merit of the selected individuals (LINDGREN and MULLIN, 1997).

\section{Results and Discussion}

\section{Effect of the input parameters}

Among the four input parameters (narrow-sense heritability, dominant to additive genetic variance ratio, patch size and gradient of site variation), heritability had the most significant impact on the accuracy of individual tree ranking. As demonstrated in Figure 1, the coefficient of correlation between ranks of the true $(I B V t)$ and predicted $(I B V p)$ individual breeding values increased as the heritability increased regardless of experimental design, pattern of environmental variation, and magnitude of dominant genetic variance. Such a result is not surprising as we should expect that correlation between the predicted and true breeding values increases with increasing heritability and so does the correlation between their ranks (FALCONER, 1989). Moreover, even though different field designs demonstrated different ranking accuracies when environmental variation was not random, the differences became smaller as heritability increased. For traits with high heritability, accurate ranking is always expected and the use of sophisticated experimental designs becomes less important (Figure $1 b$ and $1 c$ ).

Environmental patch size showed much greater impact than environmental gradient on the accuracy of ranking individuals. Kendall's coefficient of correlation between ranks of the true and predicted breeding values increased with patch size for designs with small plots, while the opposite was true for those with large plots (Figure 2). This trend was even more pronounced at lower heritabilities. With fixed patch size $(\alpha=6)$, however, no clear trend between the correlation coefficient and the slope of the environmental gradient was observed in any of the simulated experimental designs (Figure 3). These results are also somewhat intuitive as, unlike patch variation which is two dimensional (along both $\mathrm{X}$ and $\mathrm{Y}$ co-ordinates), gradient variation is one dimensional; it can be effectively removed from experimental error by both STP and MTP designs as long as the multiple-tree row plots are arranged along the gradient direction (which is the case in this simulation study, and in most tree breeding testing programs). Therefore, the impacts of gradient variation on ranking individuals should be relatively small regardless of plot size. When environmental variation is patchy, or two dimensional, designs with small plots are more effective in sampling such variation in both directions and removing it out of the experimental error. As patch size increases, more environmental variation can be removed by small plots (or small plot designs become more effective) while the opposite is true for large plots.

As demonstrated in Figures 1,2 and 3, the estimated correlations between $I B V t$ and $I B V p$ ranks tended to be weaker for all simulated designs with a dominant to additive genetic variance ratio of $30 \%$ versus 0 . However, all the findings with respect to the impacts of heritability and environmental variation patterns on the accuracy of individual tree ranking, as discussed above, are generally the same.

\section{Effectiveness of the field designs}

The percentages of the total true breeding values of the top 100 individuals ranked based on $I B V p$, over that of the top 100 individuals ranked based on $I B V t$, for the five different test designs, with medium and large environmental patch sizes and gradient slopes, and 30\% dominant to additive genetic variance ratio are presented in Table 1. It is clear that incomplete block design with single-tree plots (ICB1) provides the highest "realized" percent gains and therefore is the most effective design for ranking individuals at low to medium heritabilities $\left(h^{2} \leq 0.25\right)$, while complete block design with 2tree plots (RCB2) becomes better at medium to high heritabilities $\left(h^{2}>0.25\right)$. Complete block design with singletree plots (RCB1) tends to be superior to RCB2 at low heritabilities $\left(h^{2}<0.15\right)$ but its superiority disappears as heritability increases. The large ten-tree row plot design (RCB10) is the least effective in all the simulated scenarios.

Differences in efficiency or accuracy of ranking individuals for forward selection among different field designs are attributable to their differences in the precision of predicting individual breeding values. As mentioned earlier, the breeding value of an individual consists of two components corresponding to the family and within-family additive genetic effects. The following may serve as a simplified expression of the breeding value of an individual in terms of heritability: 


$$
\sigma_{D}^{2} / \sigma_{A}^{2}=\mathbf{0}
$$
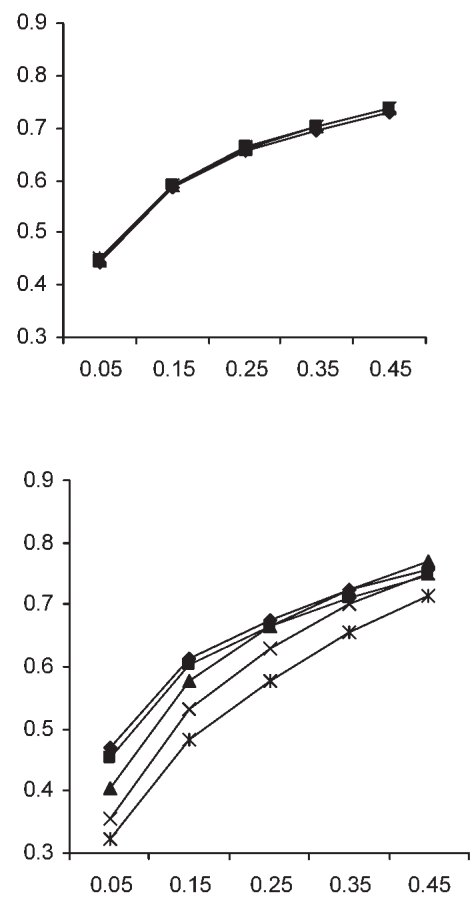

(b)

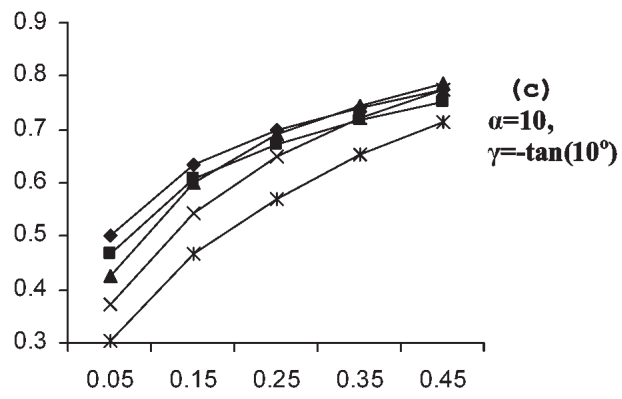

(a) $\boldsymbol{\alpha}=\mathbf{1}$,
$\gamma=\mathbf{0}$

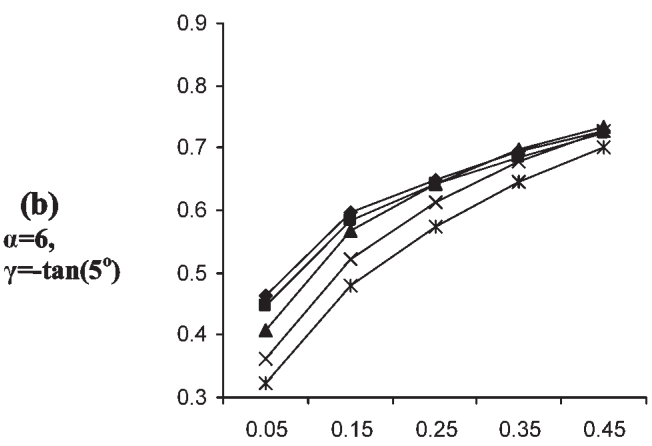
$\sigma_{D}^{2} / \sigma_{A}^{2}=\mathbf{0 . 3 0}$
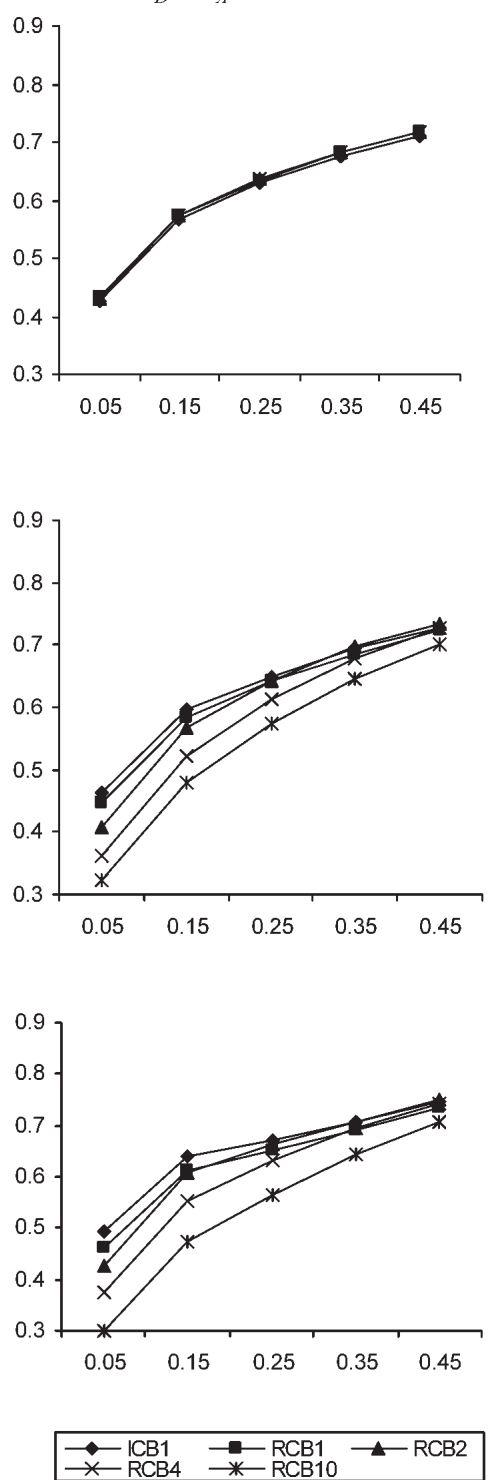

Figure 1. - Effect of heritability (X-axis) on the accuracy of individual tree ranking as measured by Kendall's coefficient of correlation (Y-axis) between the true and estimated ranks.

$$
I B V p=h_{f}^{2}\left(\bar{X}_{f}-\mu\right)+h_{w}^{2}\left(X_{i}-\bar{X}_{f}\right),
$$

where,

$$
h_{f}^{2}=h^{2} \frac{1+(n-1) 0.5}{1+(n-1) 0.5 h^{2}},
$$

and

$$
h_{w}^{2}=h^{2} \frac{0.5}{1-0.5 h^{2}} \quad(\text { FALCONER, 1989) }
$$

$h^{2}, h_{f}^{2}$ and $h_{w}^{2}$ are the narrow-sense, family and withinfamily heritability, respectively, $\mu$ is the population mean, $\bar{X}_{f}$ is the family mean, $X_{i}$ is the phenotypic value of the individual, and $n$ is the number of individuals tested per family. $h_{f}^{2}$ and $h_{w}^{2}$ roughly serve as weights to the observed family and within-family effects in predicting individual breeding values. From Equations 6 and 7, the relationship between $h_{f}^{2}, h_{w}^{2}$ and $h^{2}$ is illustrated in
Figure 4. As $h^{2}$ decreases the ratio, $h_{f}^{2} / h^{2}$, increases. As $h^{2}$ approaches zero, $h_{f}^{2} / h^{2}$ approaches $n+1$. In other words, when narrow-sense heritability is low, precise estimation of family effect rather than within-family effect is more important for precise prediction of individual breeding values. Single-tree plots maximize the number of micro-sites sampled with a given number of trees per family. Therefore, STP designs (ICB1 and RCB1) provide the most precise estimates of family means and consequently the most precise predictions of individual breeding values. Since ICB1 further reduces within-block variance due to reduced block size, it is even more efficient than RCB1 for predicting individual breeding values and therefore more effective for ranking individuals for forward selection in such situations. As the narrow-sense heritability increases, however, the difference between $h_{f}^{2}$ and $h^{2}{ }_{w}$ decreases and eventually estimation of both family and within-family effects 
$\sigma_{D}^{2} / \sigma_{A}^{2}=\mathbf{0}$
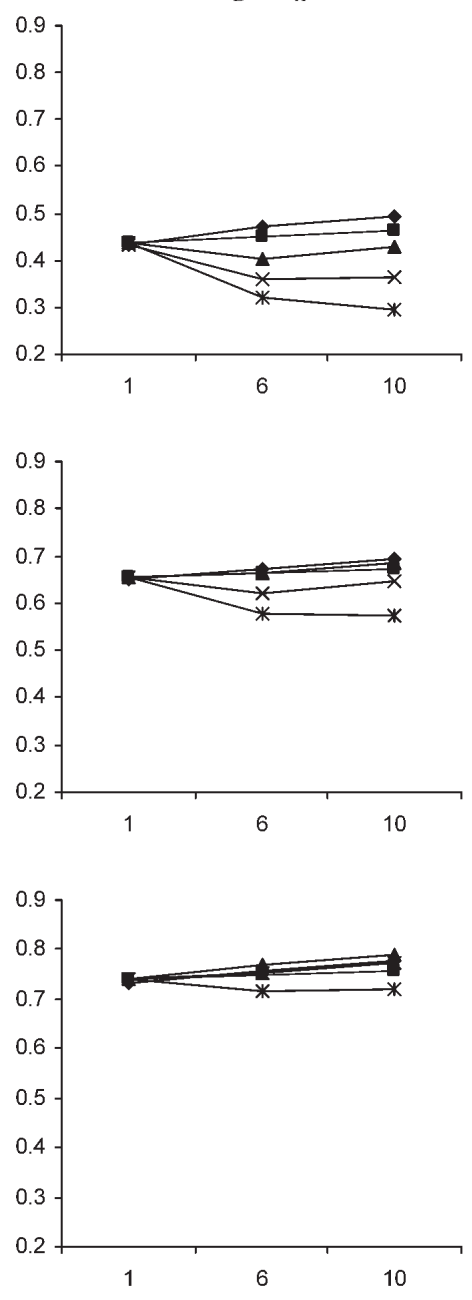

$\sigma_{D}^{2} / \sigma_{A}^{2}=\mathbf{0 . 3 0}$

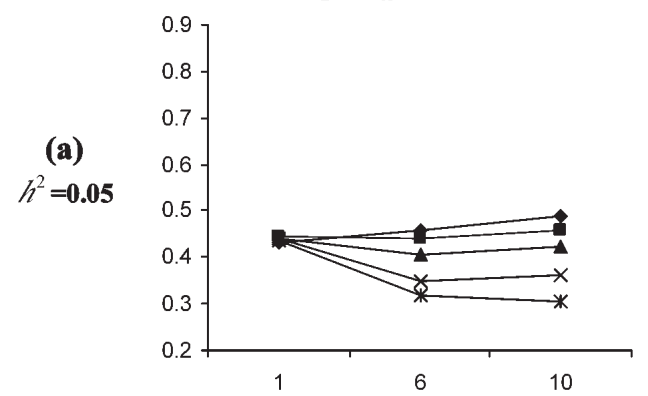

(b)
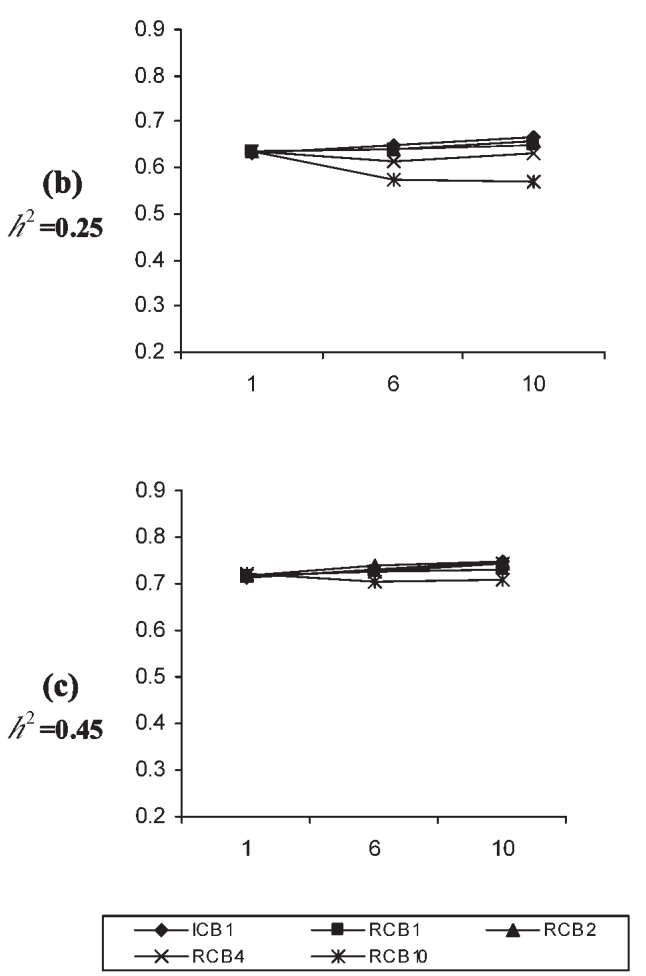

Figure 2. - Effect of environmental patch size (X-axis) on the accuracy of individual tree ranking as measured by Kendall's coefficient of correlation (Y-axis) between the true and estimated ranks $\left(\gamma=-\tan \left(5^{\circ}\right)\right)$.

becomes equally important. Unlike STP, MTP offers the opportunity of making micro-site adjustment to the phenotypic value of an individual and therefore provides superior estimate of the within-family effect. Since MTP designs sacrifice the precision of estimating family effect while improving the estimation of within-family effect, their efficiencies in predicting individual breeding values or their effectiveness in ranking individuals is determined by the net effect on the precision of estimating the two complementary components. The randomized complete block design with two-tree plots (RCB2) maximizes environmental sampling with a given number of trees tested per family, among all MTP designs, while providing the necessary information that can not be provided by STP designs for micro-site adjustments to the individual phenotypic values. Accordingly, RCB2 replaces ICB1 as the most effective design for ranking individuals when heritability increases to medium and higher. It should be pointed out that mortality was not considered in the present simulation study. It has been previously shown that the statistical efficiencies of STP designs (particularly, ICB1) decrease as mortality increases (Fu et al., 1999b). The impact of mortality on the efficiency of RCB2 design should be smaller since fewer missing plots would be generated by the same level of mortality. Loss in statistical efficiency due to mortality will decrease the precision of breeding value predictions, which in turn will lower the accuracy of individual ranking. Therefore, RCB2 may have an advantage over ICB1 at heritabilities lower than what were indicated in this study in real situations.

CoRNELIUs (1994) reported that 'average' heritabilities for traits that are commonly targeted in many forest tree breeding programs are from 0.2 to 0.3 for growth and form traits and greater for wood quality. With such heritabilities and the spatial patterns of site variation that are most commonly observed in coastal Douglas-fir progeny test trials in $\mathrm{BC}\left(\alpha=6, \gamma=-\tan \left(5^{\circ}\right)\right.$ ) (FU et al., 1999a), ICB1, RCB1 and RCB2 demonstrated comparable effectiveness in ranking individuals for forward 
selection (Figures 2 and 3, Table 1). In those cases, the choice of a particular design may depend on other factors such as the size and configuration of the potential test site, the anticipated mortality, establishment and long-term maintenance.

Another interesting observation is that with a given heritability, the most effective design is always most effective across all the simulated scenarios with respect to the pattern of site variation and the magnitude of dominant genetic variance. Thus, heritability of the target trait is the most important determinant for choosing the design of maximum effectiveness for ranking individuals in progeny testing. However, this is not to say that site selection is not important. As demonstrated in Table 1, different site variation patterns result in different effectiveness for the same design and the same heritability. Knowledge of site variation patterns may also help to determine other design parameters such as block

$$
\sigma_{D}^{2} / \sigma_{A}^{2}=\mathbf{0}
$$
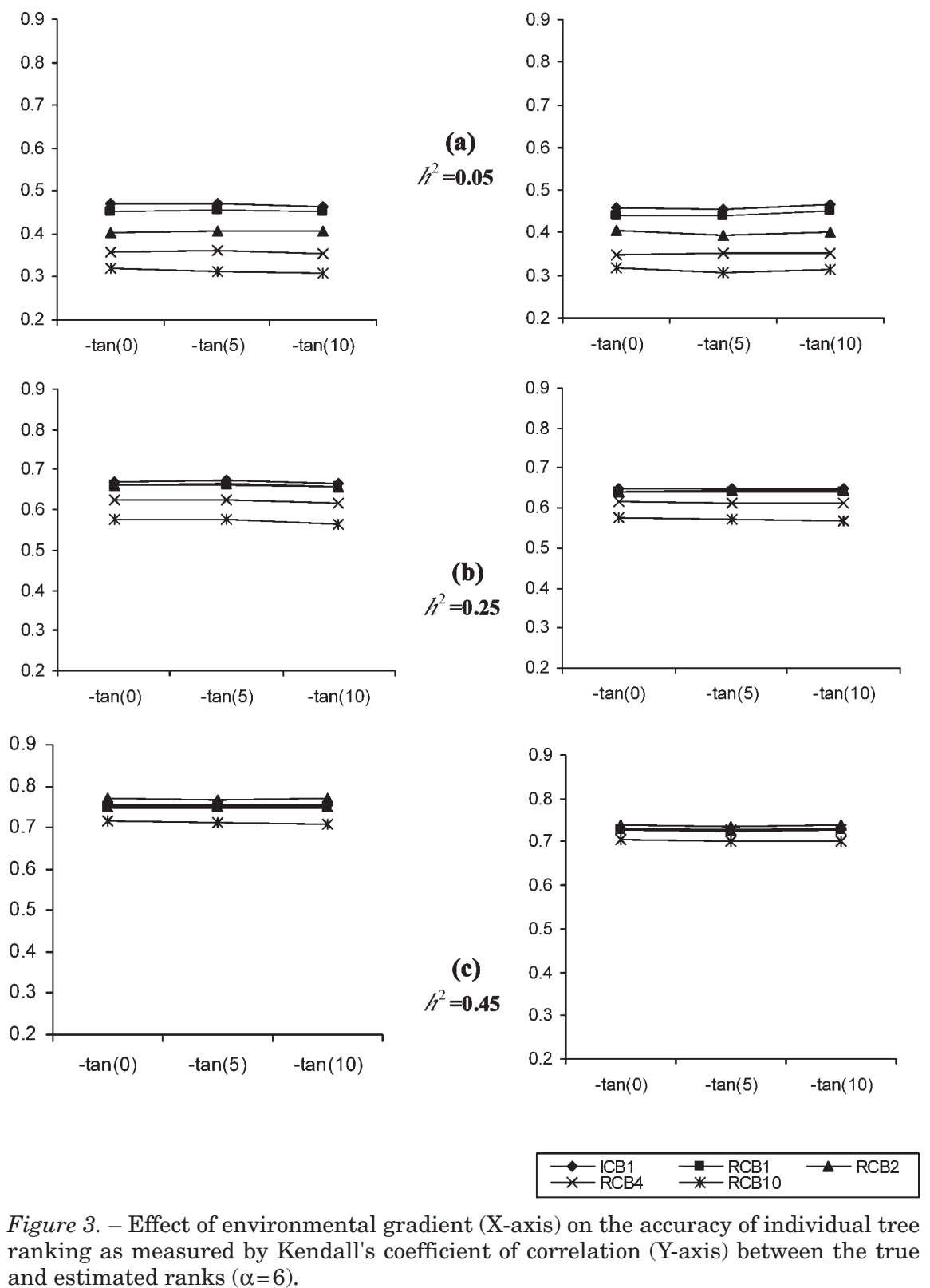

Figure 3. - Effect of environmental gradient (X-axis) on the accuracy of individual tree ranking as measured by Kendall's coefficient of correlation (Y-axis) between the true and estimated ranks $(\alpha=6)$. (a)

shape (i.e., square, row or column blocks) and block size in case of incomplete block designs (Fu et al., 1999c).

\section{Conclusions}

Simulation results from the present study indicate that the underlying heritability of the trait has the most significant impact on the accuracy of ranking individuals and is the most important factor to consider for choosing field designs in progeny testing. Incomplete block design with single-tree plots is most effective at low heritabilities while complete block design with 2tree plots appears to be the best at higher heritabilities. Large row plot designs are among the least effective in all the simulated scenarios. For traits with medium heritabilities, the statistical efficiencies of ICB1, RCB1 and RCB2 in ranking individuals are comparable. In those situations, the choice of a particular design may largely

$$
\sigma_{D}^{2} / \sigma_{A}^{2}=\mathbf{0 . 3 0}
$$

\section{(b)}


Table 1. - Percentages of the total IBVt of the top 100 individuals ranked based on IBVp over that of the top 100 individuals ranked based on IBVt for different designs with various heritability, site variation patterns and a dominant to additive genetic variance ratio of 0.30 .

\begin{tabular}{|c|c|c|c|c|c|}
\hline $\begin{array}{l}\text { Heritability/ } \\
\text { Site Variation } \\
\text { Patterns }\end{array}$ & $\mathrm{ICB}$ & $\mathrm{RCB} 1$ & $\mathrm{RCB} 2$ & $\mathrm{RCB} 4$ & $\mathrm{RCB} 10$ \\
\hline \multicolumn{6}{|l|}{$h_{i}^{2}=0.05$} \\
\hline$\alpha=6, \quad \gamma=-\tan \left(5^{\circ}\right)$ & 45.3 & 43.9 & 40.0 & 35.1 & 31.3 \\
\hline$\alpha=10, \gamma=-\tan \left(10^{\circ}\right)$ & 49.2 & 46.4 & 42.5 & 37.8 & 29.1 \\
\hline \multicolumn{6}{|l|}{$h_{i}^{2}=0.15$} \\
\hline$\alpha=6, \quad \gamma=-\tan \left(5^{\circ}\right)$ & 59.3 & 57.8 & 56.9 & 52.1 & 47.3 \\
\hline$\alpha=10, \gamma=-\tan \left(10^{\circ}\right)$ & 64.1 & 61.0 & 61.2 & 55.2 & 47.4 \\
\hline \multicolumn{6}{|l|}{$h_{i}^{2}=0.25$} \\
\hline$\alpha=6, \quad \gamma=-\tan \left(5^{\circ}\right)$ & 65.4 & 64.6 & 64.5 & 61.1 & 58.1 \\
\hline$\alpha=10, \gamma=-\tan \left(10^{\circ}\right)$ & 67.0 & 65.1 & 66.7 & 63.7 & 56.8 \\
\hline \multicolumn{6}{|l|}{$h_{i}^{2}=0.35$} \\
\hline$\alpha=6, \quad \gamma=-\tan \left(5^{\circ}\right)$ & 69.4 & 68.6 & 69.6 & 67.8 & 64.7 \\
\hline$\alpha=10, \gamma=-\tan \left(10^{\circ}\right)$ & 71.1 & 69.3 & 71.2 & 69.7 & 64.3 \\
\hline \multicolumn{6}{|l|}{$h_{i}^{2}=0.45$} \\
\hline$\alpha=6, \quad \gamma=-\tan \left(5^{\circ}\right)$ & 72.7 & 72.7 & 73.6 & 72.8 & 70.0 \\
\hline$\alpha=10, \gamma=-\tan \left(10^{\circ}\right)$ & 74.8 & 73.5 & 75.2 & 74.8 & 70.9 \\
\hline
\end{tabular}

depend on other factors such as the size and configuration of the potential test site, the anticipated mortality, establishment and long-term maintenance.

\section{References}

ConkLe, M. T. (1963): The determination of experimental plot size and shape in loblolly and slash pines. North Carolina State University, School of Forestry, Tech. Rep. No. 17.

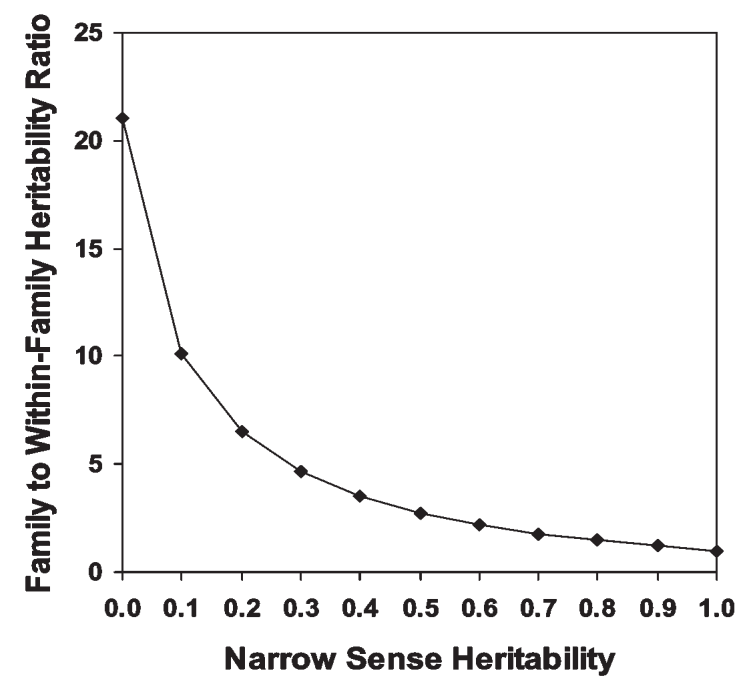

Figure 4. - Relationship between narrow sense heritability $\left(h^{2}\right)$ and the ratio of family to within-family heritabilities $\left(h_{f}^{2} / h^{2}{ }_{w}\right)$.
Cornelius, J. (1994): Heritabilities and additive genetic coefficients of variation in forest trees. Can. J. For. Res. 24: 372-379.

FALCONER, D. S. (1989): Introduction to Quantitative Genetics, $3^{\text {rd }}$ ed. Longman Scientific and Technical. New York.

Fu, Y. B., G. P. Y. Clarke, A. D. Yanchuk and G. NAMKOONG (1998): Incomplete block designs for genetic testing: statistical efficiencies of estimating family means. Can. J. For. Res. 28: 977-986.

Fu, Y. B., A. D. YANCHUK and G. NAMKOONG (1999a): Spatial patterns of tree height variations in a series of Douglas-fir progeny trials: implications for genetic testing. Can. J. For. Res. 29: 714-723.

Fu, Y. B., A. D. YANChuK, G. NAMkoOng and G. P. Y. ClARKE (1999b): Incomplete block designs for genetic testing: statistical efficiencies with missing observations. For. Sci. 45: 374-380.

Fu, Y. B., A. D. Yanchuk, G. Namkoong and G. P. Y. CLARKE (1999c): Incomplete block designs for genetic testing: some practical considerations. Can. J. For. Res. 29: $1871-1878$.

LINDGREN, D. and T. J. MulLIN (1997): Balancing gain and relatedness in selection. Silvae Genet. 46: 124-129.

LiBBy, W. J. and C. C. Cockerham (1980): Random noncontiguous plots in interlocking field layouts. Silvae Genet. 29: 183-190.

SAS InstituTE INC. (1995): SAS User Guide. Version 6.03 ed. Cary, NC.

SokAL, R. R. and F. J. RohlF (1981): Biometry, $2^{\text {nd }}$ ed. W. H. Freeman and Company, San Francisco. 
van BuiJtenen, H. P. (1983): Progeny test design and analysis. Proc. South. Tree Improv. Conf. 17: 161-170.

WRIGHT, J. W. (1976): Introduction to forest genetics. Academic Press Inc., New York, N.Y.
XIANG, B. and B. LI (2003): Best linear unbiased prediction of clonal breeding values and genetic values from full-sib mating designs. Can. J. For. Res. 33: 2036-2043.

\title{
Genetic Parameters and Strategies for Genetic Improvement of Stiffness in Radiata Pine
}

\author{
By S. KumaR ${ }^{1), *)}$, H. S. DungeY ${ }^{1)}$ and A. C. Matheson ${ }^{2)}$
}

(Received $13^{\text {th }}$ October 2005)

\begin{abstract}
The two main objectives of this study were: (1) to determine how early is it possible to undertake selection to improve the stiffness of corewood; (2) to determine if the selection based on corewood stiffness could also improve outerwood stiffness, and vice versa. Breastheight data from two progeny trials of Pinus radiata D. Don were used. In the first trial (age 30 years), data on Silviscan predicted stiffness $(\mathrm{MoE})$ was obtained for each growth ring on each core sample from 50 open-pollinated families. In the second trial (age 14 years), data on static-bending MoE was obtained using clearwood sticks $(300 \times 20 \times 20 \mathrm{~mm})$ cut from each tree from 18 control-pollinated families. MoE varied from $3.5 \mathrm{GPa}$ in rings $1-5$ to about $17 \mathrm{GPa}$ in rings $21-25$. Coefficients of variation of corewood and outerwood MoE were about $20-30 \%$ and $15-20 \%$ respectively. Estimates of narrowsense heritability for $\mathrm{MoE}$ were generally higher $(0.50-0.70)$ in the corewood compared with the outerwood (0.15-0.30). Early selection for MoE could yield substantial gain in corewood MoE but only small gains, if any, in outerwood MoE (especially for rings 21-30). Estimated genetic correlations between density and stiffness appeared moderate in the corewood zone, but high in the outerwood zone. Selection based on density (using 5-mm cores) and acoustic stiffness (using standing tree tools), assessed at age 6-7 years, appeared to be a good option to improve both corewood and outerwood stiffness.
\end{abstract}

Key words: Stiffness, sound velocity, wood density, heritability, breeding strategy, genetic correlation, age-age correlation, early selection, Pinus radiata.

\section{Introduction}

Interest in the genetic improvement of wood properties of radiata pine (Pinus radiata D. Don) has increased to an all-time high. This is mainly driven by the aim to increase the recovery of higher-grade of sawn timber. Wood stiffness, measured as modulus of elasticity (MoE), is a key property of structural sawn timber. Low

\footnotetext{
1) Ensis-Genetics, Private Bag 3020, Rotorua, New Zealand.

2) Ensis-Genetics, PO Box E4008, Kingston ACT 2604, Australia.

*) Corresponding author: Tel.: +64 7343 5899, Fax: +64 73480952 , Email: Satish.Kumar@forestresearch.co.nz
}

stiffness is an important limitation of radiata pine timber, especially when grown on the short rotations (25-30 years), which affects the quality and performance under certain specific usage. Such rotations result in a high proportion of corewood (the first 10 rings or so from the pith) leading to an associated reduction in wood quality (Cown et al., 1987; Cown et al., 1999) because corewood has poorer wood quality for most applications. Various direct, indirect and surrogate tests can be used to estimate wood stiffness (BETHGE and MATtheCK, 1998; XIPING et al., 2000; JAYAWICKRAMA, 2001; KumAR et al., 2002; LinTSTRÖM et al., 2002; MATHESON et al., 2002):

(1) Stiffness can be measured directly on sawn lumber; however, this would be very expensive for ranking genotypes, and trees cut down for testing would no longer be available for breeding or other work unless clonally replicated.

(2) Small clear wood specimens (taken from the tree at breast-height or from inside the felled stem) are another option, and specimens of $300 \times 20 \times 20 \mathrm{~mm}$ have been used routinely in New Zealand (e.g. BIER and BRITTON, 1999).

(3) SilviScan, a wood microstructure analyser (Evans, 2003), can be used to obtain predicted MoE for different rings by scanning strips cut from 12 -mm increment cores.

(4) Stiffness can be measured indirectly through acoustic tools, such as HITMAN, which measures resonance in logs (HARRIS and ANDREWS, 1999), or IML Hammer, which measures the elapsed time of propagation of an acoustic stress wave in standing trees (ANONYMUs, 2001).

Timber stiffness of radiata pine was identified as a priority for the New Zealand radiata pine breeding programme in 1997 (SoRENSSON, unpublished), with highranking parents (for growth and form traits) being subsequently ranked for stiffness and various other wood properties. As a result, a large number of parents of the New Zealand radiata pine breeding population have been ranked for breeding values, based on acoustic stiffness (KUMAR, 2004). HITMAN measurements require felled logs, which means that selected trees will not be available for propagation as forward selections or re- 